Review Article

\title{
An update on neuraxial opioid induced pruritus prevention
}

\begin{abstract}
The itching or pruritus is a secondary effect very annoying that appears after the neuraxial administration (epidural or intrathecal) of opioid drugs. Sometimes it can even be more unpleasant that the own pain in or by itself. Both, either prevention or treatment remains a challenge in the clinical practice of care for these patients. A wide variety of medications with different mechanisms of action have been used and focused in its prevention and treatment, with widely varying results. The objective of this article is to review the literature and summarize the current evidence of the mechanisms and pharmacological treatments available to handle pruritus induced by spinal opioids. The source of articles of this review was obtained through PubMed, Medline and Scopus until 2016. The most useful drugs are opioid mu antagonists, as naloxone, and mixed opioids kappa agonists /mu antagonists, as nalbuphine and butorphanol, the latter being able in addition to maintaining the analgesia. They have also shown some effectiveness, but to a lesser degree, from receptor antagonists of the serotonin 5-HT3, as ondasetron, administered prophylactically, and the antagonists of dopaminergic receptors D2, as dehidrobenzoperidol. Finally sub anesthetic low dose of propofol and oral mirtazapine have been used with medium results.
\end{abstract}

Keywords: complications, epidural, itching, neuraxial opioids, post-operative, pruritus, spinal, mu and kappa opioid receptors
Volume 6 Issue 3 - 2016

Borja Mugabure Bujedo

Department of Anesthesiology and Pain medicine, Donostia University Hospital, Spain

Correspondence: Borja Mugabure Bujedo, Department of Anesthesiology and Pain medicine, Donostia University Hospital, San Sebastián, Paseo del Dr. Beguiristain II7, 200I4, Spain, Tel +34 943007000, Fax +34 943007233, Email mugabure@yahoo.es

Received: October 27, 2016 | Published: November 25, 2016
Abbreviations: NO, neuraxial opioids; MOP, MU-opioid receptor; KOP, kappa-opioid receptor; ITM, intrathecal morphine; IV, intravenous; PONV, postoperative nausea and vomiting

\section{Introduction}

The incidence of pruritus after intrathecal administration of opioids varies between $30-100 \%$ without large differences depending on the drug involved, being higher in the pregnant woman and in mixtures with adrenaline. Usually, this adverse effect occurs on the face and trunk and is mediated by a mechanism that is unknown, although the modulation of the serotonin system seems to play an important role. The release of histamine does not appear to be involved, and there must be a central mechanism since the use of antagonists such as naloxone and naltrexone reverses the clinic. Antihistamines are used with the aim of decreasing the scratching. The serotonin receptor antagonist 5HT-3, ondansetron (4-8mg iv), has been shown to be effective in the prevention and treatment of this symptom, as well as the subhipnótic dose of propofol $(10 \mathrm{mg}$, with or without infusion $30 \mathrm{mg} / 24 \mathrm{~h}$ ). Finally butorphanol and nalbuphine (4mg iv) that appears to demonstrate a greater effectiveness and should be consider the firstlime treatment. ${ }^{1-5}$

\section{Discussion}

\section{Clinical use of neuraxial opioids}

Opioids have always been considered the best option in clinical practice for the treatment of severe postoperative pain. However, the spinal administration of an opioid drug does not always guarantee selective action and segmental analgesia in the spine. This fact is due to partial reuptake to blood systemic circulation reaching brain receptors. Recent evidence from clinical studies indicates that bioavailability in the spinal cord bio phase is negatively correlated with liposolubility, which is higher for hydrophilic opioids, than for lipophilic ones. Actually, clinical guidelines recommend using a mixture of local anesthetic plus a strong opioid to improve the global analgesic effect, minimize adverse effects and improve the overall patient's satisfaction. Moreover, an opioid alone like morphine can be administered to provide a long period of postoperative analgesia for $24 \mathrm{~h}$, or even $48 \mathrm{~h}$ when an extended release epidural formulation is used. In this narrative review, practical information for correct spinal opioid selection is provided to help the physicians a better approach to postoperative multimodal spinal analgesia. ${ }^{6-13}$ Morphine could be the main opioid involved in the presence of NO adverse effects such as pruritus, urinary retention, PONV and delayed respiratory depression. ${ }^{14,15}$

\section{Mechanism of neuraxial opioid induced pruritus}

The exact mechanism of neuraxial opioid induced pruritus is not clear. Many mechanisms have been postulated, but there is no single mechanism that can explain all cases. The mechanisms involved include: ${ }^{16,17}$

a. The activation of the "itching center" in the central nervous system.

b. Medullary activation: The medullary dorsal horn activation and antagonism of inhibitory transmitters

c. The modulation of the serotonergic pathway

d. The theory that links the pain and itching.

It seems that pain and itching are transmitted by the same population of sensory neurons, such as small nerve unmielinizated fibers (C fibers) and the release of prostaglandins (PGE1 and PGE2). All this improves the transmission of $\mathrm{C}$ fibers in the central nervous system, which powers the pruritus. ${ }^{18}$ A high density of subtype 5-hydroxytryptamine 3 receiver (5-HT3) and mu receptors are present in the superficial layers of the dorsal horn and in the core of the spinal tract of the trigeminal nerve in the bone marrow. The spinal nucleus 
of the trigeminal located superficially in the marrow is an integrating center of sensory information of the face and an area known as the "center of itching." The cephalic migration of NO toward this "center of itching" and the activation of the 5-HT3 receptors by opioids can play a role in the generation of neuroaxial opioid-induced pruritus. ${ }^{19}$

Opioids also can induce itching at the spinal level by the secondary neurons in the lamina I spinal-thalamic stretch of the dorsal horn. The neurons of wide range activation in the dorsal horn inhibit these spinal neurons itching. If this inhibition is weakened by the opioids, neurons are activated and cause itching without peripheral stimulation. The activation of the marrow and the production of itching, it is observed, in particular, by the activation of the mu opioid receptors (MOR), although the opioid receptors kappa- (KOR) suppress the itching. ${ }^{20}$

\section{Prevention and treatment of neuraxial opioid induced pruritus}

Opioid receptor antagonists: The receptor $\mu$ is responsible for the modulation of pain and some side effects, in particular the pruritus and nausea or vomiting. Moreover, MOR antagonist drugs should be the first line treatment for all opioid-induced side effects.

Nalmefene, originally known as nalmetrene, is a mu opioid receptor antagonist developed in mid-1970, which has mainly been used in the US for the treatment of alcoholism and opioid overdose. It has also been investigated for the treatment of pruritus induced by NO in primates with good results. $\mathrm{KO}$ et al. ${ }^{21}$ demonstrated that a single prophylactic dose of this drug $(32 \mu \mathrm{g} / \mathrm{kg})$ were effective to reduce the itching and also intrathecal morphine-induced analgesia. It developed a clear deviation to the right of the dose/response curve in primates studied against both clinical parameters. This fact demonstrated to the authors that the therapeutic window is really very close between the production of analgesia and pruritus, both mediated by mu receptors. The pre-treatment with clocinamox, a selective antagonist mu, inhibited scratching induced by spinal opioids in primates, but neither the antagonism $\kappa$-opioid (binaltorfimina) nor the antagonists delta (naltrindol) produce this effect. This would explain the fundamental antipruritic role of the antagonists to mu receptors. ${ }^{22}$ Accordingly, many studies have evaluated the efficacy of naloxone, naltrexone and methyl naltrexone in the prevention of pruritus, but results were observed variables. On the basis of existing data, a low dose, intravenously or in infusion, naloxone has the greatest evidence for the prevention of $\mathrm{NO}$ induced pruritus $\mathrm{ON}$ in the adult. A continuous infusion produces less fluctuation of concentrations than naloxone bolus injections and compensates for the relatively short half-life of naloxone..$^{23}$ It seems that an intravenous dose of 0.25 to $1 \mathrm{mcg} / \mathrm{kg} / \mathrm{h}$ is the most effective dose without affecting the analgesia. A systematic review of Kjellberg and Tramèr in the year 2001 with 834 patients $^{24}$ concluded that intravenous naloxone was an effective drug in the treatment of pruritus and nausea induced by $\mathrm{NO}$, without increasing the pain score, and that higher doses than $2 \mathrm{mcg} / \mathrm{kg} / \mathrm{h}$ were more likely to lead to the reversal of the analgesia and therefore are not recommended. But a review at the University of York and their system DARE (Database of Abstracts of reviews of Effects) concluded that due to the heterogeneity of the studies included in this review and the high percentage of methodological errors, the authors not considered reliable the results. ${ }^{25}$

On the other hand, another recent meta-analysis showed the effectiveness of naloxone in the prevention and treatment of the adverse effects of the opioid drugs. After a selective selection, only six studies were included in the paper, which examined the effects of naloxone in opioid-induced pruritus (4 of them via spinal and 2 track systemic). The analysis indicated that there was significant heterogeneity between the included studies ( $\mathrm{I} 2=60.3 \%, \mathrm{p}=0.027$ ). Therefore, meta-analysis was based on the random effects model. The results indicated that the rate of incidence of pruritus induced by opioids was significantly lower in the group of naloxone versus the control group $(\mathrm{RR}=0.252$, IC $95 \%=0.137-0.464, \mathrm{P}=0.000) .{ }^{26}$

However, under the conditions of a recent study, a single dose of $12 \mathrm{mg}$ of methyl of subcutaneous methyl naltrexone did not reduce the overall severity or the incidence of pruritus among an obstetric population after receiving, fentanyl $15 \mathrm{mcg}$ and $100 \mathrm{mcg}$ of ITM, during spinal anesthesia for cesarean delivery. Prophylactic treatment with a peripheral mu antagonist mu was ineffective against the intrathecal morphine-induced pruritus in the peripartum period, although a small clinical effect could not be excluded. ${ }^{27}$ In this regard, similar results were found in a study in orthopedic surgery. Subcutaneous administration of methylnaltrexone was not effective to reduce the postoperative urinary retention and pruritus, but reduced the incidence of PONV after intrathecal bupivacaine and morphine. ${ }^{28}$

Mixed opioid receptor agonist/Antagonist: The mixed opioid agonists kappa-antagonist mu as nalbuphine or partial agonists $\mu$ (MOR) and $\mathrm{k}(\mathrm{KOR})$ as butorphanol and pentazocine, have a great potential to mitigate the adverse effects by MOR and to improve the analgesic effects of opioids on KOR. The experimental studies have demonstrated that either the antagonists MOR or the agonists KOR are effective in the relief of itching induced by the intrathecal morphine induced in primates. ${ }^{29}$ The effectiveness of nalbuphine, butorphanol and pentazocine has been studied with positive results. ${ }^{30,31}$ Tamdee et al. ${ }^{32}$ conducted a randomized trial to study the effectiveness of pentazocine for the treatment of pruritus associated with the intrathecal injection of morphine and concluded that the pentazocine at a dose of $15 \mathrm{mg}$ is superior to ondansetron $4 \mathrm{mg}$ for the treatment of intrathecal pruritus induced by ITM.

In a recent systematic review on nalbuphine published in $2016,^{33}$ ten studies (1129 patients) met all the inclusion criteria, 9 of which were randomized controlled trials and 1 clinical case report. The incidence of pruritus was higher among the patients who received $\mathrm{NO}$ on that those with the intravenous route. Nalbuphine provided a greater effectiveness in the treatment of opioid induced pruritus, compared with placebo, control, or other pharmacological agents such as diphenhydramine, naloxone, and propofol. There was no attenuation of the analgesia or increase of the sedation with low doses of nalbuphine treatment; $25 \%$ to $50 \%$ of the dose to treat the pain, i.e. 2.5 to $5 \mathrm{mg}$ versus $10 \mathrm{mg}$ intravenously. In addition, nalbuphine was associated with a reduction of nausea or vomiting, and with the reduction of respiratory depression. Author's conclusions were that nalbuphine is superior to treat itching opioid induced in patients receiving No for acute pain related to surgery or childbirth. They therefore recommended that the nalbuphine should be used as a first line treatment for opioid induced pruritus. The author explained the lack of transfer of these findings to the clinical practice due to the absence of a clinical guide based on the evidence in this field, the fact counter-intuitive of dealing with the adverse effects of an opioid with another drug opioid, use outside technical sheet (offlabel) of nalbuphine for treatment of pruritus, and even the largest price compared to other alternatives in the North American market. However, this drug has not demonstrated the same effectiveness in child population, ${ }^{34}$ although their lower diffusion to breast milk than other opioids make it an attractive option in the obstetric population. ${ }^{33}$

In the year 2013 it was published a systematic review on the effectiveness of the butorphanol in the No induced pruritus. ${ }^{35}$ The 
main results of Sixteen trials ( $\mathrm{n}=795$ patients) were analyzed. By intravenous and epidural routes, butorphanol reduced pruritus with a RR of 0.22 (95\% CI 0.10 to 0.45 ) and RR 0.24 (95\% CI 0.16 to 0.36 ), respectively by both routes. The use of butorphanol epidural reduced the number of patients seeking treatment of rescue for the itching (RR $0.57 ; 95 \%$ CI 0.41 to 0.81 ). The butorphanol diminished the intensity of postoperative pain at the four, eight and 12hours, with the differences in VAS score (visual analogue scale) of CI -0.29 (95\% CI -0.52 to -0.05$),-0.30$ ( $95 \%$ CI -0.56 to -0.04$)$ and -0.23 (95\% CI- 0.46 to -0.01), respectively. However, the intravenous epidural butorphanol did not reduce the incidence of postoperative nausea and vomiting (PONV) (RR 0.35; 95\% CI: 0.19 to 0.66). Butorphanol did not increase the respiratory depression (RR $0.71 ; 95 \% \mathrm{CI} 0.31$ to 1.63 ), or the drowsiness (RR0.71; 95\% CI 0.22 to 2.37 ) or dizziness (RR $2.45 ; 95 \%$ CI 0.35 to 17.14 ). In conclusion, butorphanol administered with morphine may be an effective strategy to prevent itching due to it also reduces the intensity of the pain and PONV without increasing other side effects. Therefore, the authors concluded that this drug could be recommended to prevent the induced pruritus $\mathrm{ON}$ during the perioperative period.

5HT3 Antagonist: The exact mechanism of ondansetron to relieve the itching is unknown. Although the 5-HT3 receptors can be identified in the spinal cord of rodents and primates, there is no evidence for the co-anatomical location of the 5-HT3 receptors with the MOP in the spinal cord, or functional tests to corroborate the interaction between the receiver 5-HT3 and MOP in any of the animal models studied previously. As an example, after the administration of intrathecal morphine $(32 \mu \mathrm{g})$ gave rise to a deep sense of scratching for the itching ( $\sim 600$ scratches in $15 \mathrm{~min} /$ sampling time) in rhesus monkeys $(n=8)$ (Unpublished data of the Ko lab). Ondansetron was administered intravenously $(0,1-3,2 \mathrm{mg} / \mathrm{kg})$ approximately $2 \mathrm{~h}$ after the subjects received intrathecal morphine. Within these doses evaluated in the present study, the ondansetron was ineffective to mitigate the pruritus induced by the intrathecal morphine. A higher dose of ondansetron $(10 \mathrm{mg} / \mathrm{kg})$ caused extrapyramidal reactions in monkeys (involuntary contractions, stiffness in both legs and with spasm of the Muscles extender) that led to the end of the experiments. Because of this, the author does not support the routine use of these drugs. ${ }^{20}$

The 5-HT3 receptors are located at the dorsal horn of the spinal cord and spinal tract of the trigeminal nerve. It is for that reason that the interaction between opioids and 5-HT3 receptors can play a decisive role in the generation of the induced pruritus by NO. The 5-HT3 antagonists, such as ondansetron, granisetron, dolasetron, have been used prophylactically to prevent the opioid induced pruritus. A systematic review of 15 randomized controlled trials $(n=1337)^{36}$ indicated that the prophylactic treatment with a iv bolus dose of receptor antagonists 5-HT3 can provide a significant decrease in the incidence and the score of the intensity of pruritus after administration of $\mathrm{NO}$, in particular when using intrathecal morphine but not lipophilic opioids. It was also found a significant decrease in the use of rescue medication for the treatment of pruritus. Dosages for receptor antagonists of 5-HT3 ondansetron used were iv. $4 \mathrm{mg}$, $8 \mathrm{mg}$ or $0.1 \mathrm{mg} / \mathrm{kg}$. Other receptor antagonists $5-\mathrm{HT} 3$ studied were Tropisetron $(5 \mathrm{mg})$, granisetron $(3 \mathrm{mg})$, and dolasetron (12.5mg). More specifically, studying the population of pregnant women undergoing cesarean section under spinal anesthesia with intrathecal morphine, it was found in a systematic review with meta-analysis, that although the prophylactic treatment with receptor antagonists 5-HT3 were ineffective in reducing the incidence of pruritus, this drug significantly reduced the severity and the need for the treatment of this adverse effect, the incidence of nausea and vomiting, as well as the need for rescue antiemetic drugs. It was also effective for the treatment of pruritus established..$^{37}$ Although more studies are needed, the current data suggest that the routine prophylactic use of medicines should be considered in this patient population.

In another recent meta-analysis, the antagonist of the 5-HT3 receptor mainly used in clinical practice, it was not reduced the incidence of pruritus after injection of high liposolubility opiates as fentanyl or sufentanil. The administration of prophylactic $8 \mathrm{mg}$ iv ondansetron did not decrease the incidence of pruritus but could decrease the need for rescue medication, especially in specific subgroups including non-obstetric surgery care and in patients who received the drug before the administration of spinal opioids.$^{38}$ One of the assumptions is that morphine mechanisms to be less fat-soluble and has slower onset of analgesia, gives rise to a greater concentration of residual opioid in the cerebrospinal fluid and greater migration cephalic. As the peak concentration of ondansetron, occurs around 15 min, the 5-HT3 antagonists can reach 5-HT3 receptors in the spinal cord before the morphine, but not after the fat-soluble drugs. ${ }^{39}$

\section{Other drugs}

NSAIDS: Anti-inflammatory drugs (NSAIDS) have a role well recognized in postoperative pain relief. They inhibit the cyclooxygenases (COX) and decrease the formation of prostaglandins involved in the peripheral tissue inflammatory process. It has been demonstrated that iv. Tenoxicam ${ }^{40}$ and rectal diclofenac ${ }^{41}$ possess certain anti-pruriginous effects in patients receiving spinal opioids. However, Gulhas et al. ${ }^{42}$ found no decrease of pruritus with the use of lornoxicam after administration of intrathecal fentanyl. The Celecoxib, a selective COX-2, has shown variable results in studies on anti-pruriginous effects. Lee et al. ${ }^{43}$ found no reduction of pruritus with the oral use of celecoxib after the administration of morphine for intrathecal track. Their study showed no effect antipruritic or significant analgesic in a single dose of $200 \mathrm{mg}$ (administered after the birth of the baby) in the first $24 \mathrm{~h}$ after the surgery of cesarean section. However, Samimi et al. ${ }^{44}$ using $400 \mathrm{mg}$ of celecoxib by mouth $1 \mathrm{~h}$ before surgery demonstrated its effectiveness in reducing the incidence of pruritus induced by intrathecal morphine in this subgroup of patients.

In another experimental study (Unpublished data of the Ko lab) compared the effects of ketorolac and nalmefene after intrathecal morphine-induced in rhesus monkeys $(\mathrm{n}=5)$. Any of the 2 drugs, ketorolac $(10 \mathrm{mg} / \mathrm{kg})$ or nalmefene $(32 \mu \mathrm{g} / \mathrm{kg})$ was administered intravenously approximately $2 \mathrm{~h}$ after the subjects received intrathecal morphine $(32 \mu \mathrm{g})$. In this experimental context, intravenous nalmefene, but not ketorolac, significantly decreased the response of scratching against the pruritus. Based on these results, the NSAIDS cannot be useful therapeutic agents to treat itching. According to its author, ${ }^{20}$ it seems unlikely that prostaglandins play an important role as mediators of the itching associated with NO.

Antihistamines: Though morphine may trigger the release of histamine from mast cells, clinical studies have indicated that the antihistamines are not effective in the relief of pruritus induced by NO. ${ }^{45}$ The pharmacological studies in non-human primates also found that an antihistamine such as diphenhydramine, in a wide range of doses could not attenuate the intrathecal morphine-induced pruritus. ${ }^{22}$ In addition, other agonists of MOP as fentanyl and alfentanil do not stimulate the release of histamine, while they evoke pruritus/ scratching in humans and non-human primates. ${ }^{46}$ As the taquifilaxia develops rapidly in the local response to histamine, its role is minimal in the core processes of the induced pruritus. 
H1 blockers are therefore of little or no effect on the central pruritus induced by NO. In spite of this, the antagonists of the H1 receptors of first generation, such as diphenhydramine or hydroxyzine may produce a sedative effect, which could sometimes be useful in patients with pruritus. The proposed mechanism would be disruption on the whole cycle of itching and scratching, while providing a better quality of sleep, which is necessary, but they are not very effective in reducing the severity of the itching. ${ }^{4,20}$

Propofol: Propofol has been used for the treatment and prevention of pruritus. It exerts its antipruritic action through the inhibition of the transmission from the dorsal horn of the spinal cord. Many studies have been carried out with the sub-hypnotic dose of propofol, ranging from $10 \mathrm{mg}$ bolus until $30 \mathrm{mg}$ during $24 \mathrm{~h}$ in infusion, but the results were contradictory. ${ }^{4,20}$

Dopaminergic antagonists D-2: Droperidol and alizaprida have also been used for the treatment of opioid induced pruritus. Both are potent antagonists of the dopamine D2. Droperidol also has a weak activity anti-5-HT3. In the study of Horta et al. ${ }^{45}$ on 300 women undergoing cesarean section, the subgroup that received droperidol intravenously showed the lowest prevalence of pruritus in comparison with placebo and also in comparison with propofol, alizaprida, and promethazine. Metoclopramide, another antagonist of dopamine D2 receptor has proved to be ineffective in this regard.

Mirtazapine: Mirtazapine is a new antidepressant that selectively blocks 5-HT2 and 5-HT3 receptors. Mirtazapine has a unique pharmacological profile, since apart from increasing noradrenergic and serotoninergic neurotransmission; it may exercise its antidepressant and anti-nociceptive action through the system $\kappa$-opioid dependent. Its anti-pruritus activity was first described by Davis et al. ${ }^{47}$ when it was demonstrated in this study, its capacity to reduce the induced pruritus by intrathecal morphine. Sheen et al. ${ }^{48}$ studied Mirtazapine to reduce intrathecal morphine-induced pruritus and concluded that the oral preoperative administration of mirtazapine $30 \mathrm{mg}$ decreased the incidence, delayed the onset time, reduce the severity, and shortened the duration of pruritus. Secondly, mirtazapine may act in the cerebral cortex to reduce the perception of pruritus. Thirdly, mirtazapine presented a strong antihistamine effect. From the pharmacokinetic point of view mirtazapine has another advantage over the receptor antagonists 5-HT3 of first generation. The peak concentration of mirtazapine is reached $2 \mathrm{~h}$. after a single dose and the elimination halflife varies from $20 \mathrm{~h}$ to $40 \mathrm{~h}$ allowing the drug could cover the start and the whole range of duration of pruritus. The most common observed adverse effects were sedation, somnolence and dry mouth.

\section{Conclusion}

A large variety of drugs have been evaluated in the treatment of NO induced pruritus. Among them, many drugs are included, such as gabapentin, the antagonists of the dopamine D2, propofol, mirtazapine, and dexamethasone. All of them have been discussed in recent years in review articles, but results were inconclusive from a very limited number of clinical studies. Because these drugs have not been widely studied in non-human primates, there is little chance of moving the results on the potential pharmacological antagonism of these drugs to clinical trials. On the whole, these pharmacological studies indicate that Rhesus monkeys can serve as a surrogate species for human beings in the preclinical studies to identify effective treatments for spinal opioid induced pruritus. Despite this, the accumulated pharmacological evidence supports the antagonists of the MOP (mu opioid receptor) and mixed partial agonists mu and kappa (KOP/MOP) options as the most effective treatment for spinal opioid induced pruritus. Indeed, partial or mixed agonist/antagonist opioids like intravenous or epidural butorphanol (1-3mg) and nalbuphine (4mg iv) appears to demonstrate the greatest effectiveness, either on prevention or treatment, and should be considered as firstline treatment of pruritus induced by NO.

The rest of therapeutic options that act in the non-opioid ligands, such as the 5-HT3 antagonists (ondansetron), antihistamines, and NSAIDS, have not shown sufficient effectiveness to be considered as first-line treatment in this field. The release of histamine does not appear to be involved, and there must be a central mechanism since the use of antagonists such as naloxone, but not peripheral antagonist like metilnaltrexone, reverses the pruritus clinic. Antihistamines are used only with the aim of decreasing the scratching. Serotonin receptor antagonists 5 HT-3, as ondansetron (4-8mg iv), have shown a moderate degree of effectiveness in the prevention and treatment of this symptom, especially after intrathecal morphine in obstetric patients but not under lipophilic opioids such as fentanyl or sufentanil, as well as low dose propofol (20mg).

\section{Acknowledgments}

To my family and to the members of the Pain Unit from Donostia University Hospital.

\section{Conflicts of interest}

The authors declare there are no conflicts of interest.

\section{Funding}

None.

\section{References}

1. Ballantyne JC, Loach AB, Carr DB. Itching after epidural and spinal opiates. Pain. 1988;33(2):149-160.

2. Szarvas S, Harmon D, Murphy D. Neuraxial opioid-induced pruritus: A review. J Clin Anesth. 2003;15(3):234-239.

3. Kam PC, Tan KH. Pruritus: Itching for a cause and relief? Anaesthesia. 1996;51(12):1133-1138.

4. Kamal Kumar, Sudha Indu Singh. Neuraxial opioid-induced pruritus: An update. J Anaesthesiol Clin Pharmacol. 2013;29(3):303-307.

5. Reich A, Szepietowski JC. Opioid-induced pruritus: An update. Clin Exp Dermatol. 2010;35:2-6.

6. Bujedo BM. Spinal opioid bioavailability in postoperative pain. Pain Pract. 2014;14(4):350-364.

7. Wang JK, Nauss LA, Thomas JE. Pain relief by intrathecally applied morphine in man. Anesthesiology. 1979;50(2):149-151.

8. Behar M, Magora F, Olshwang D, et al. Epidural morphine in treatment of pain. Lancet. 1979;313(8115):527-529.

9. Bernards CM. Understanding the physiology and pharmacology of epidural and intrathecal opioids. Best Pract Res Clin Anaesthesiol. 2002;16(4):489-505.

10. Mugabure Bujedo B, González Santos S, Uría Azpiazu A, et al. Up to date in clinical management of neuraxial opioids for the treatment of postoperative pain. Rev Soc Esp Dolor. 2012;19(2):72-94.

11. Bujedo BM, Santos SG, Azpiazu AU. A review of epidural and intrathecal opioids used in the management of postoperative pain. $J$ Opioid Manag. 2012;8(3):177-192.

12. Chaney MA. Side effects of intrathecal and epidural opioids. Can $J$ Anaesth. 1995;42(10):891-903. 
13. Rathmell JP, Lair TR, Nauman B. The role of intrathecal drugs in the treatment of acute pain. Anesth Analg. 2005;101(5 Suppl):S30-S45.

14. The American Society of Anesthesiology (ASA). An updated report by the American Society of Anesthesiologists Task Force on neuraxial opioids. Practice Guidelines for the prevention, detection and management of respiratory depression associated with neuraxial opioid administration. Anesthesiology 2009;110:218-230.

15. Gulhas N, Erdil FA, Sagir O, et al. Lornoxicam and ondansetron for the prevention of intrathecal fentanyl-induced pruritus. J Anesth. 2007;21:159-163.

16. Ganesh A, Maxwell LG. Pathophysiology and management of opioidinduced pruritus. Drugs. 2007;67(16):2323-2333.

17. Schmelz M. Opioid-induced pruritus. Mechanisms and treatment regimens. Anaesthesist. 2009;58(1):61-65

18. Andrew D, Craig AD. Spinothalamic lamina I neurons selectively sensitive to histamine: A central neural pathway for itch. Nat Neurosci. 2001;4(1):72-77.

19. Liu XY, Liu ZC, Sun YG, et al. Unidirectional cross-activation of GRPR by MOR1D uncouples itch and analgesia induced by opioids. Cell. 2011;147(2):447-458.

20. Ko MC. Neuraxial opioid-induced itch and its pharmacological antagonism. Handb Exp Pharmacol. 2015;226:315-335.

21. Ko MC, Naughton NN. An experimental itch model in monkeys: characterization of intrathecal morphine-induced scratching and antinociception. Anesthesiology. 2000;92(3):795-805.

22. Ko MC, Song MS, Edwards T, et al. The role of central mu opioid receptors in opioid-induced itch in primates. $J$ Pharmacol Exp Ther. 2004;310(1):169-176.

23. Miller JL, Hagemann TM. Use of pure opioid antagonists for management of opioid-induced pruritus. Am J Health Syst Pharm. 2011;68(15):1419-1425.

24. Kjellberg F, Tramèr MR. Pharmacological control of opioid-induced pruritus: A quantitative systematic review of randomized trials. Eur $J$ Anaesthesiol. 2001;18(6):346-357.

25. Murphy JD, Gelfand HJ, Bicket MC, et al. Analgesic efficacy of intravenous naloxone for the treatment of postoperative pruritus: a metaanalysis. J Opioid Manag. 2011;7(4):321-327.

26. He F, Jiang Y, Li L. The effect of naloxone treatment on opioid-induced side effects: A meta-analysis of randomized and controlled trails. Medicine (Baltimore). 2016;95(37):e4729.

27. Paech M, Sng B, Ng L, et al. Methylnaltrexone to prevent intrathecal morphine-induced pruritus after Caesarean delivery: a multicentre, randomized clinical trial. Br J Anaesth. 2015;114(3):469-476.

28. Zand F, Amini A, Asadi S, et al. The effect of methylnaltrexone on the side effects of intrathecal morphine after orthopedic surgery under spinal anesthesia. Pain Pract. 2015;15(4):348-354.

29. Ko MC, Lee H, Song MS, et al. Activation of kappa-opioid receptors inhibits pruritus evoked by subcutaneous or intrathecal administration of morphine in monkeys. J Pharmacol Exp Ther. 2013;305(1):173-179.

30. Lawhorn CD, McNitt JD, Fibuch EE, et al. Epidural morphine with butorphanol for postoperative analgesia after cesarean delivery. Anesth Analg. 1991;72(1):53-57.

31. Charuluxananan S, Kyokong O, Somboonviboon W, et al. Nalbuphine versus propofol for treatment of intrathecal morphine-induced pruritus after cesarean delivery. Anesth Analg. 2001;93(1):162-165.
32. Tamdee D, Charuluxananan S, Punjasawadwong Y, et al. Randomized controlled trial of pentazocine versus ondansetron for the treatment of intrathecal morphineinduced pruritus in patients undergoing cesarean delivery. Anesth Analg. 2009;109(5):1606-1611.

33. Jannuzzi RG. Nalbuphine for Treatment of Opioid-induced Pruritus: A Systematic Review of Literature. Clin J Pain. 2016;32(1):87-93.

34. Nakatsuka N, Minogue SC, Lim J, et al. Intravenous nalbuphine 50 microg $\mathrm{x} \mathrm{kg} \mathrm{(-1)} \mathrm{is} \mathrm{ineffective} \mathrm{for} \mathrm{opioid-induced} \mathrm{pruritus} \mathrm{in} \mathrm{pediatrics.}$ Can J Anaesth. 2006;53(11):1103-1110.

35. Du BX, Song ZM, Wang K, et al. Butorphanol prevents morphineinduced pruritus without increasing pain and other side effects: a systematic review of randomized controlled trials. Can J Anaesth. 2013;60(9):907-917.

36. Bonnet MP, Marret E, Josserand J, et al. Effect of prophylactic 5-HT3 receptor antagonists on pruritus induced by neuraxial opioids: A quantitative systematic review. Br J Anaesth. 2008;101(3):311-319.

37. George RB, Allen TK, Habib AS. Serotonin Receptor Antagonists for the Prevention and Treatment of Pruritus, Nausea, and Vomiting in Women Undergoing Cesarean Delivery with Intrathecal Morphine: A Systematic Review and Meta-Analysis. Anesth Analg. 2009;109(1):174-182.

38. Prin M, Guglielminotti J, Moitra V, et al. Prophylactic Ondansetron for the Prevention of Intrathecal Fentanyl- or Sufentanil-Mediated Pruritus: A Meta-Analysis of Randomized Trials. Anesth Analg. 2016;122(2):402-409.

39. Pernia A, Calderón E, Calderón Pla E, et al. Ondansetron in the treatment of the pruritus associated with the spinal infusion of opiates. Rev Esp Anestesiol Reanim. 2000;47(9):425-426.

40. Colbert S, O'Hanlon DM, Chambers F, et al. The effect of intravenous tenoxicam on pruritus in patients receiving epidural fentanyl. Anaesthesia. 1999;54(1):76-80.

41. Colbert S, O'Hanlon DM, Galvin S, et al. The effect of rectal diclofenac on pruritus in patients receiving intrathecal morphine. Anaesthesia. 1999;54(10):948-952.

42. Gulhas N, Erdil FA, Sagir O, et al. Lornoxicam and ondansetron for the prevention of intrathecal fentanyl-induced pruritus. $J$ Anesth. 2007;21(2):159-163.

43. Lee LH, Irwin MG, Lim J, et al. The effect of celecoxib on intrathecal morphine-induced pruritus in patients undergoing Caesarean section. Anaesthesia. 2004;59(9):876-880.

44. Samimi S, Davari Tanha F, Malekian M. A blinded study using celecoxib for prevention of morphine induced pruritus in patients undergoing cesarean section. J Fam Reprod Health. 2011;2(5):35-39.

45. Horta ML, Morejon LC, da Cruz AW, et al. Study of the prophylactic effect of droperidol, alizapride, propofol and promethazine on spinal morphine-induced pruritus. Br J Anaesth. 2006;96(6):796-800.

46. Ellis DJ, Millar WL, Reisner LS. A randomized double-blind comparison of epidural versus intravenous fentanyl infusion for analgesia after cesarean section. Anesthesiology. 1990;72:981-986.

47. Davis MP, Frandsen JL, Walsh D, et al. Mirtazapine for pruritus. J Pain Symptom Manage. 2003;25(3):288-291.

48. Sheen MJ, Ho ST, Lee $\mathrm{CH}$, et al. Prophylactic mirtazapine reduces intrathecal morphine-induced pruritus. Br J Anaesth. 2008;101(5):711715 